\title{
Anne-Marie BRISEBARRE, Liliane KUCZYNSKI, (dirs.), La Tabaski au Sénégal. Une fête musulmane en milieu urbain
}

Paris, Karthala, 2009, 466 p.

André Julliard

\section{(2) OpenEdition}

\section{Journals}

Édition électronique

URL : http://journals.openedition.org/assr/22658

DOI : 10.4000/assr.22658

ISSN : $1777-5825$

Éditeur

Éditions de l'EHESS

Édition imprimée

Date de publication : 31 décembre 2010

Pagination : 9-242

ISBN : 9782713223013

ISSN : 0335-5985

\section{Référence électronique}

André Julliard, « Anne-Marie brisebarRe, Liliane kuczynskı, (dirs.), La Tabaski au Sénégal. Une fête

musulmane en milieu urbain », Archives de sciences sociales des religions [En ligne], 152 | octobredécembre 2010, document 152-22, mis en ligne le 17 mai 2011, consulté le 21 septembre 2020. URL : http://journals.openedition.org/assr/22658 ; DOI : https://doi.org/10.4000/assr.22658

Ce document a été généré automatiquement le 21 septembre 2020

(c) Archives de sciences sociales des religions 


\section{Anne-Marie BRISEBARRE, Liliane KUCZYNSKI, (dirs.), La Tabaski au Sénégal. Une fête musulmane en milieu urbain}

Paris, Karthala, 2009, 466 p.

André Julliard

\section{RÉFÉRENCE}

Anne-Marie BRISEBARRE, Liliane KUCZYNSKI, (dirs.), La Tabaski au Sénégal. Une fête musulmane en milieu urbain, Paris, Karthala, 2009, 466 p.

1 L'étude de la fête de la Tabaski, nom wolof de l'Aïd el-Kébir, résulte d'une recherche ethnographique franco-sénégalaise, entre le Laboratoire d'Anthropologie sociale (Anne-Marie Brisebarre et Liliane Kuczynski), l'Institut Fondamental d'Afrique Noire (Pampa Demba Fall, géographe, Virginia Tiziana Bruzzone, anthropologue) et l'Université Cheik Anta Diop avec Mame Yassine Sarr et Abdou Thiam, étudiants en socio-anthropologie. Rejointe par Ndiawar Kane (géographe), l'équipe se réunit autour d'un programme de recherches centré moins sur la pratique sacrificielle du mouton que sur: «Les enjeux culturels, sociaux et économiques de la fête de la Tabaski en milieu urbain sénégalais» qui s'inscrit dans «l'appel 2000 à proposition en science sociale» du ministère des Affaires étrangères.

2 Les deux premiers chapitres dressent le décor général de la fête et, en premier lieu, celui du religieux. La Tabaski (douzième mois du calendrier musulman) commémore le sacrifice d'Isaac ordonné à Abraham/Ibrahim par Yahvé. Reconnaissant la pleine soumission du patriarche à Sa loi, Dieu suspend le geste sanglant au moment suprême en lui faisant apporter, par l'ange Gabriel, un «mouton céleste» comme victime sacrificielle de substitution. Yahvé veut éprouver à la fois la réalité de la crainte et la 
radicalité de l'obéissance qu'Il inspire à Abraham. L'épreuve scelle l'alliance avec la descendance d'Abraham qui fonde le monothéisme commun aux trois religions révélées.

3 Isaac est l'enfant unique et légitime que Sara, l'épouse alors très âgée d'Abraham, met au monde après avoir été «visitée» par Dieu (Gen. 21,1-2). Un certain nombre de textes savants et de récits populaires arabes remplacent Isaac par Ismaël, son demi-frère aîné conçu par la servante Agar en accord avec Sara qui pensait ne plus pouvoir enfanter (Gen. 16).

4 Sans entrer dans l'analyse de ce dossier ouvert dans un ouvrage précédent sur le sacrifice en islam (Pierre Bonte, Anne-Marie Brisebarre, Altan Gokalp, (dirs.), Sacrifices en islam. Espaces et temps d'un rituel, CNRS Éditions, Paris, 1999), nous retiendrons avec les auteurs que ce débat n'a pratiquement pas d'échos dans la majorité des maisons sénégalaises d'aujourd'hui. Dans le temps de la fête, les familles s'attachent plutôt à valoriser l'histoire commune en partageant avec leurs voisins chrétiens une part du mouton sacrifié. Dans ce sens, et en nous appuyant sur des observations répétées en Casamance et à Dakar, il semble que l'on peut faire un parallèle entre les fêtes de la Tabaski et de la Pâque qui, hors de tout débat doctrinal, sont propices à des questionnements sur les pratiques religieuses respectives des musulmans et des catholiques. Pourquoi les seconds ne commémorent-ils pas le geste d'Abraham? Qu'estce que la Pâque? L'approche de ces échanges informels, spontanés, conviviaux bien que variables selon l'appartenance confrérique, permettrait d'interroger «la cohabitation harmonieuse des religions» que le Sénégal affiche comme l'un des caractères de sa démocratie.

Mais le mouton reste le vrai souci économique et familial de la Tabaski. Anne-Marie Brisebarre, Papa Demba Fall et Ndiawar Kane étudient minutieusement les races ovines élevées au Sénégal et dans la sous-région, la commercialisation (grossistes, circuit de vente, éleveur-vendeurs occasionnel, etc.) dans les foirails urbains ou ruraux, les critères de sélection et les modalités d'achat de l'animal. En laissant la partie ethnozoologique, on retiendra que le «mouton de Tabaski» doit être un grand bélier robuste, aux cornes bien développées et au pelage blanc. Plutôt rare sur le marché, un tel animal est réservé aux clients les plus riches et l'acheteur ordinaire doit limiter ses ambitions à un mouton aux caractères les plus proches de l'idéal type.

Concernant la pratique sacrificielle, le choix du mouton (victime) pose la question du rapport au sacrifiant. Les auteurs constatent unanimement qu'aucun lien n'unit animal et sacrifiant à l'exception du prestige d'en être le propriétaire (certains d'entre eux lavent ou font laver le mouton avant de procéder au sacrifice) même si c'est pour les quelques jours qui séparent l'achat de l'abattage. Le caractère exceptionnel de ce statut économique, inaccessible en temps ordinaire pour la majorité des Sénégalais, construitil le véritable lien entre la victime et le sacrificateur que rompt le sacrifice le jour venu? $\mathrm{Au}$ quotidien, les soucis du propriétaire sont plus matériels car il doit veiller à ce que l'animal ne soit ni volé ni atteint de maladie: la perte financière, souvent irremplaçable, anéantit la fête religieuse et familiale.

7 Pourtant, il y a quelques attaches décelables lorsque chaque auteur note sur son terrain qu'un mouton blanc apporte la bénédiction divine sur la famille alors qu'un noir porte malheur et doit être refusé ou, en dernier ressort, entrer de nuit dans la maison. On peut, également, admettre que dans les familles modestes, le père qui en plus de trouver l'argent, acquiert un mouton à la robe claire, accroît son prestige de chef de 
famille non seulement auprès de ses femmes et enfants, mais aussi de la rue et de son quartier. Il a su se "débrouiller» autant sur le plan économique que symbolique en ramenant «un peu de chance» à la maison!

8 Mais il est vrai que ces pratiques ne suffisent pas à nouer entre victime et sacrifiant ces liens d'identité nécessaires au processus sacrificiel. Elles renvoient plus sûrement aux systèmes de savoirs, plus vastes, de protection et de guérison de la personne dans lesquels entrent certains éléments organiques prélevés sur la dépouille encore chaude du mouton. Les femmes, principalement, confectionnent des remèdes qui se conservent d'une Tabaski à l'autre. Virginia Tiziana Bruzzone en énumère un certain nombre parmi lesquels le sang séché mélangé à de l'eau contre les otites (Toucouleur), la poudre de langue grillée sur les abcès (Sérère et Lébou) et, beaucoup plus répandue, le cartilage thyrö̈dien prélevé par le sacrifiant dans la coupure de la gorge qui, une fois séché, s'emploie en collier contre les angines.

9 Si nous mettons de côté l'offrande de viande aux rabs chez les Lébou, simplement évoquée dans l'ouvrage, il faut remarquer qu'en définitive le mouton de la Tabaski fournit peu de matière spécifique à la fabrication d'objets apotropaïques ou thérapeutiques. On peut faire le parallèle avec le sacrifice dans les religions de terroir où rien n'est prélevé sur l'animal-victime qui est soigneusement surveillé jusqu'à sa complète consommation. La comparaison permet de penser que, d'un côté, la charge sacrificielle n'est pas suffisamment exceptionnelle pour développer des usages familiaux «mystiques» alors que, de l'autre, dans le sacrifice non musulman, elle est trop puissante pour laisser quiconque s'approprier une parcelle de cette force (?)

10 Seules les cornes du bélier, comme le note l'auteure, font l'objet de ce désir d'appropriation et leur traitement varie d'une pratique de l'islam à l'autre. Dans le sud du Sénégal, les familles Diola islamisées et Manding les gardent ou les vendent à des marabouts pour être «chargées en médecines» de protection (ou autre). Par contre, les tidjanes, à l'islam plus intellectuel, veillent scrupuleusement à les enterrer profondément avec les viscères de l'animal pour éviter toute convoitise étrangère.

11 Ensuite viennent neuf chapitres qui nous permettent de suivre pas à pas les auteurs sur leurs terrains ethnographiques:

12 - Dakar, la capitale au peuplement dense et cosmopolite; - Saint-Louis, à l'embouchure du fleuve Sénégal dans l'océan Atlantique, est l'ancienne capitale coloniale où se côtoient Bambara (protestant), Wolof et Toucouleur (musulmans); - Touba, la ville sainte de la puissante confrérie mouride qui devient un pôle économique, culturel et politique dépassant largement les frontières du Baol (Sénégal central) et même du Sénégal grâce à une forte émigration (Italie, États-Unis, France) des talibés (élèves, disciples) extrêmement fidèles au khalife général des mourides. L'actuel président de la République du Sénégal est l'un des talibés les plus engagés dans la diffusion de cet islam confrérique: «USA: Wade explique le mouridisme aux étudiants d'Utah», Bitimrew.net, 27 septembre 2010; - Tambacounda (Sénégal oriental) où les Peul organisent le commerce du mouton avec le Mali.

13 Chacun d'eux procède aux mêmes observations: marché local du mouton, sacrifice et dépeçage de l'animal dans une ou deux familles appartenant plutôt à la classe aisée sénégalaise (commerçants, enseignants, fonctionnaires), partage et offrande de la viande aux parents, voisins, obligés et aux plus démunis, repas familial et visites de courtoisie. Loin d'être lassante, la répétition entraîne le lecteur dans la fête comme s'il entendait entre les pages, les bêlements des moutons, les négociations sur le marché 
aux bestiaux, les bruits parfois musicaux des commerces de rues, l'agitation et l'effervescence des familles, les prières à la mosquée.

Du nord au sud, la Tabaski fatigue beaucoup les Sénégalais dont l'immense majorité doit se débrouiller pour trouver toute la dépense nécessaire à la fête. L'achat du mouton représente la part la plus importante du budget et chacun recourt à de multiples stratégies de négoce. Parmi les plus courantes, citons l'endettement personnel; le groupement d'achat au sein des administrations, établissements scolaires ou entreprises; l'élevage à domicile d'un ou deux moutons: le second, revendu à la Tabaski, amortit les frais d'entretien et constitue un capital pour renouveler l'opération lors de la prochaine fête; l'endettement désespéré du buki (la hyène) qui consiste à se procurer une liquidité financière en achetant par mensualité un appareil électroménager, par exemple, pour le revendre immédiatement moins cher au comptant.

Autre poste de dépenses incompressibles qui, le plus souvent, incombent entièrement au mari: couvrir la famille en vêtements neufs. L'obligation sociale est suffisamment pesante pour que dans certaines populations (Diola, Manding), l'homme n'hésite pas à se priver d'un nouveau boubou pour que ses épouses et enfants ne risquent pas sarcasmes et railleries. Les femmes doivent, également, se faire coiffer et cette charge leur revient si elles sont financièrement autonomes. Aussi, en ville et au village, l'obligation de "paraître» peut se comprendre comme «un rite social de dignité» par lequel on honore la famille, la parenté et, peut être, plus qu'un rang social, une place dans la société. En effet, la ténacité à se procurer coûte que coûte des habits neufs coutume dénoncée aujourd'hui pour sa futilité - peut se comprendre comme une façon de célébrer plus fondamentalement «la vie», «d'être encore en vie», dans une société où la précarité menace sans cesse la personne. Cette célébration se relève, également, dans les souhaits de se revoir à la prochaine Tabaski qui se formulent couramment au cours des «visites de courtoisie» constitutives du rituel festif que, toute la journée, on rend aux parents, amis, pauvres et malades.

Il faut que tout soit "changé en beau» à la maison puisque, outre qu'elle doit être nettoyée de fond en comble, les familles aisées font repeindre quelques pièces et renouveler les rideaux des portes et fenêtres. Pour les hommes, les nouveaux habits ne suffisent pas, il faut «débarrasser son cœur» des fautes commises depuis la dernière Tabaski en échangeant le pardon avec les personnes rencontrées et visitées ce jour-là. Tout se passe comme si on «jette» symboliquement les scories du temps écoulé depuis la dernière Tabaski, pour marquer le passage à un nouveau cycle annuel dans les corps et l'espace de vie quotidien.

Les auteurs remarquent que parmi les fêtes sénégalaises, religieuses et laïques (fête de l'indépendance), la Tabaski est sans doute le moment où s'exprime collectivement et de manière la plus intense, le sentiment d'appartenir à une même culture et une même société. Du point de vue de l'ethnologie de la fête, on pourrait dire qu'elle réifie l'unité sociale moins par des excès ou débordements de comportements que parce qu'elle magnifie le savoir de la débrouille qui, une fois encore, permet de vaincre le mal de survie auquel est journellement confrontée la majorité des Sénégalais. L'ouvrage invite, alors, à s'interroger sur l'impor-tance de la Tabaski qui, au Sénégal, éclipse Tamkharit (Achoura) marquant la nouvelle année alors qu'elle est considérée, notamment chez les tidjanes, comme la fête majeure du calendrier musulman. D'ailleurs, au travers du sacrifice du mouton, il existe au moins un lien matériel entre les deux fêtes: un ou deux 
tibias sont mis à sécher jusqu'à leur utilisation comme condiment dans le couscous de Tamkharit.

Le prélèvement de ces os prend place dans un scénario sacrificiel qui est le même de Dakar à Tambacounda. Le jour où se fait le même sacrifice dans le grand pèlerinage à La Mecque, le 10 du mois de Tabaski, à la sortie de la prière du matin, l'imam autorise les sacrifices dans les maisons en égorgeant publiquement son mouton sur l'esplanade de la mosquée. En disant bismillâh («avec le nom de» ou «par la grâce de Dieu, je fais»), le chef de famille tue son mouton au-dessus d'un trou creusé dans la cour ou la rue sablonneuse pour enfouir le sang, les viscères abdominaux et les cornes. Il est impératif que le cou soit tranché en une seule fois: tout tâtonnement ou reprise du geste rend caduc le sacrifice. Il transforme en quelque sorte le cadavre en "charogne» (témoignage d'un tidjane) et le rend impropre à la consommation car il laisse du sang à l'intérieur des organes (?) Puis, dans les familles aisées, il répète l'acte sanglant sur chacun des moutons offerts ou achetés par ses épouses. Tout se passe comme si le sacrifice qui est une simple recommandation de l'islam, doit «se voir» sans qu'il y ait, pour autant, obligation d'une pratique publique.

Pendant qu'il procède ou fait procéder (boucher à domicile) au dépeçage et à la découpe de l'animal, l'épouse s'empresse de faire griller le foie avec des oignons. Cuit rapidement, il permet de rompre sans délai le petit jeûne recommandé du matin, à la manière du pain et du verre de «Nescafé» qui, au coucher du soleil, coupent la journée de ramadan. Les auteurs remarquent que la symbolique liée à ce viscère («siège de l'être», «minimum vital»: 185-186) n'est pas très assurée en milieu musulman et doit varier en fonction des conceptions de la personne dans les populations concernées. Cependant, et contrairement au sacrifice dans les religions de terroir, il semble que ce repas soit une continuité de la fête et non du processus sacrificiel (?)

Après ce petit déjeuner, l'épouse partage le mouton en trois parties: l'une réservée aux repas de la fête et la deuxième conservée dans le congélateur domestique ou, en louant la place, dans celui d'une connaissance. La troisième partie est destinée à être distribuée d'abord aux parents lorsqu'ils résident à proximité immédiate de la maison. Au-delà de la multitude des variantes familiales, confrériques et régionales, le choix des morceaux de viande semble obéir à quelques conventions parentales comme le gigot ou l'épaule destinés aux sœurs du mari, les filets aux sœurs du père du mari ou de l'épouse, ou encore, le cimier réservé à la mère de la femme. L'épouse qui vit dans la famille de son mari, montre le respect qu'elle doit aux tantes et belles-sœurs, surtout si elles sont âgées et économiquement faibles.

21 Les autres parts de viande, crue ou cuite, sont «envoyées» sans retard aux amis, voisins, veuves, malades et pauvres du voisinage immédiat. Il s'agit tout à la fois de nettoyer l'atelier boucherie dans la cour, de s'empresser de gratifier d'estime les intimes de la famille, et de permettre à ceux qui n'ont pas de mouton, de cuisiner en temps voulu un repas familial de fête.

Dans les rues des quartiers, surtout urbains, de la classe moyenne aisée, la circulation des plateaux de viande est spectaculaire surtout le premier des trois jours de Tabaski reconnu par l'islam. Pour une même famille, la valeur morale et religieuse de l'offrande n'est pas la même selon le destinataire. On peut distinguer trois circuits: - la charité (donner), l'une des grandes valeurs morales de l'islam, n'a pas au Sénégal le même sens que «faire une charité»: d'un côté on n'attend pas de rendu ailleurs que dans le monde céleste (décision divine), dans l'autre, il s'agit en quelque sorte d'attirer sur soi la 
chance ou le bonheur; - la prestation de sociabilité parentale ou de relation d'affinité (bon voisinage, amitié ou entraide) dans laquelle le rapport donner/recevoir peut s'inscrire dans d'autres règles que la stricte réciprocité. Un plat envoyé à un ami n'attend pas obligatoirement un retour équivalent, mais sert plutôt à entretenir et fortifier la solidité de la relation; - l'escompte d'une gratification personnelle (matérielle, sociale ou symbolique) par l'offrande adressée au supérieur hiérarchique, à l'employeur, à un créditeur ou au dirigeant de la confrérie d'appartenance.

Liliane Kuczynski et Abdou Thiam étudient les stratégies de cette pratique particulièrement développée chez les mourides du Baol. Fortement centralisée et hiérarchisée, cette confrérie se charpente sur le clientélisme de milliers de talibés, d'affidés ou de commensaux de Sérigne Touba (khalife général). Le jour de la Tabaski, ils apportent le plat de viande traditionnel à leur marabout respectif mais, quelques jours avant la fête, ils peuvent lui offrir un ou plusieurs moutons vivants, voire, plus discrètement, des sommes d'argent. En retour, ils reçoivent la bénédiction de Dieu par le guide tout en se plaçant pour une sollicitation ultérieure: emploi, promotion ou attribution de terres cultivables. Mais comme seul le mouton acheté valide le sacrifice, toute la viande abattue est immédiatement redistribuée aux plus démunis, aux affidés les plus méritants, les plus fidèles ou, aussi, pour honorer les chefs d'administration en service dans le Baol. Il s'agit de montrer publiquement qu'il n'y a pas thésaurisation, mais redistribution totale des dons de la Tabaski. Plus leur circulation est ample, plus elle exprime le dynamisme, la cohésion et la puissance politico-économique de la communauté mouride. Simultanément, cette prospérité sociale et économique, abondamment diffusée par les médias sénégalais, «prouve» que la confrérie est sous la bénédiction d'Allah et, donc, dans la vérité de l'islam.

Ce compte rendu, trop bref, est conscient de n'avoir pas épuisé les apports de cette volumineuse étude, fourmillante de matériaux ethnographiques, qui questionnent les changements des rapports sociaux, des relations familiales, du sens de la fête, de la notion de personne et de la pratique de l'islam dans la société sénégalaise d'aujourd'hui. Aussi, je terminerai sur l'une de ces interrogations concernant la définition du sacrifice. L'ethnologie des religions en Afrique noire souligne que l'acte de mise à mort de l'animal «ne peut être dit sacrificiel que s'il vient défaire des liens qui sont tissés lors du processus rituel entre l'animal (cette fois victime) et un, ou plusieurs, des personnages engagés dans le rite» (Michel Cartry, dir., Sous le masque l'animal. Essais sur le sacrifice en Afrique noire, Paris, PUF, 1987: 8). On peut, alors, se demander si le sacrifice de la Tabaski ne relève pas davantage de l'abattage rituel qui participe plus de la médiation entre les hommes que de la communication avec le divin? 